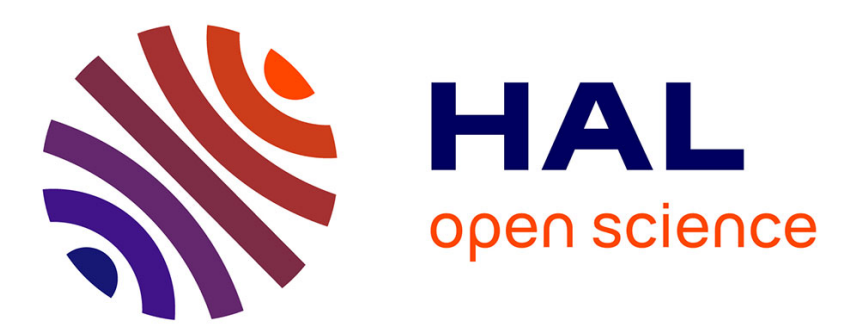

\title{
Regularidades sintáctico-semánticas en las subordinadas proposicionales del español medieval. Siglos XI al XIII
}

\author{
Marta López Izquierdo
}

\section{To cite this version:}

Marta López Izquierdo. Regularidades sintáctico-semánticas en las subordinadas proposicionales del español medieval. Siglos XI al XIII. Alexandre Veiga et Mercedes Suárez Fernández. Historiografía lingüística y gramática histórica. Gramática y léxico, 17, Iberoamericana / Vervuert, pp.19-34, 2002, Lingüística Iberoamericana. hal-01710950

\section{HAL Id: hal-01710950 \\ https://hal.science/hal-01710950}

Submitted on 8 Dec 2020

HAL is a multi-disciplinary open access archive for the deposit and dissemination of scientific research documents, whether they are published or not. The documents may come from teaching and research institutions in France or abroad, or from public or private research centers.
L'archive ouverte pluridisciplinaire HAL, est destinée au dépôt et à la diffusion de documents scientifiques de niveau recherche, publiés ou non, émanant des établissements d'enseignement et de recherche français ou étrangers, des laboratoires publics ou privés. 


\title{
REGULARIDADES SINTÁCTICO-SEMÁNTICAS EN LAS SUBORDINADAS PROPOSICIONALES DEL ESPAÑOL MEDIEVAL. SIGLOS XI AL XIII
}

\author{
Marta López Izquierdo \\ Université Lumière Lyon 2
}

En mi tesis de doctorado (López Izquierdo 2000a), propuse un conjunto de propiedades sintácticas y semánticas que permiten caracterizar los verbos de modalidad factual en español medieval (siglos XII al XV). Para ello, adopté un marco teórico que voy a conservar en este trabajo y que paso a exponer brevemente.

1. Sigo los principios de las gramáticas de base semántica, desarrolladas a partir de la noción de valencia (Tesnière 1959), combinada con la de caso: los predicados se definen así por el número de argumentos (obligatorios u opcionales) que rigen y por su naturaleza sintáctica y semántica. Utilizo concretamente el marco teórico propuesto por B. Pottier en sus trabajos de 1987 y 1992.

1.1. Pottier distingue tres niveles en la gramática de una lengua: el nivel conceptual o abstracto, el nivel de la lengua, en que se realizan las operaciones de selección de lexemas y de combinaciones sintácticas y, finalmente, el nivel discursivo, en que se manifiesta efectivamente el enunciado.

Para este autor, toda oración es el resultado de una combinación de actantes y de uno o más predicados, a partir de una base predicativa. Los ocho casos conceptuales que reconoce Pottier con validez universal son: el causal, el instrumental, el nominativo, el ergativo, el acusativo, el destinatario, el final y el locativo. Las distintas realizaciones que estas zonas casuo-conceptuales conocen en una lengua determinada constituyen los casos lingüísticos de dicha lengua. Así, para el español, por ejemplo, tanto el caso nocional ergativo como el nominativo se expresarán por medio del caso lingǘstico nominativo. Los actantes o argumentos reciben una marca casual nocional, abstracta, y otra lingüística, sintáctica. El caso nocional se representa en mayúsculas; el caso lingüístico en minúsculas. 
Los casos se organizan en tres zonas: una obligatoria; dos, participantes sugeridos; tres, la de los circunstanciales, siempre posibles.

El módulo casual de (1) representa, de acuerdo con el sistema que acabamos de describir, la estructura actancial de una oración como Ana come carne ${ }^{1}$ :

(1) $\mathrm{SN}_{\text {nom }}^{\mathrm{aERG}} \mathrm{V} \mathrm{SN} \mathrm{bACC}_{\text {acc }}$

1.2. La modalidad factual, siguiendo a Pottier, es aquella que expresa el grado de obligación de realizar la acción que se describe en el enunciado. Un verbo modal factual expresa, por consiguiente, una modificación relativa al carácter posible o necesario de la acción que figura en la proposición. Las otras dos modalidades del enunciado que mencionaré aquí son la epistémica, que se refiere al grado de adhesión del hablante a la verdad de lo enunciado, y la axiológica, que expresa por su parte un grado de apreciación sobre una escala evaluativa cualquiera: moral, estética, utilitaria...

2. A partir del comportamiento de los quince verbos estudiados ${ }^{2}$, hemos podido reconocer tres subgrupos, según el número de argumentos que exigen en construcción modal factual. Todos ellos comparten una misma característica: exigen, en su uso modal, un argumento proposicional. Utilizamos aquí el término proposición para referirnos a las oraciones completivas de sujeto o de objeto directo, por oposición al resto de las subordinadas (circunstanciales), de las que se diferencian por presentar un mayor grado de cohesión ${ }^{3}$ con respecto al verbo principal. Sin embargo, cada uno de estos grupos presenta además una serie de particularidades que configuran el módulo actancial en cada caso:

\subsection{Predicados monovalentes: poder, dever, aver...}

- V' determina las propiedades semánticas y el caso nocional del actante $a$, mientras que $\mathrm{V}$ determina su caso lingüístico. En el ejemplo (2), el

\footnotetext{
${ }^{1}$ Otras convenciones utilizadas: representamos con letras $(a, b, c \ldots)$ los argumentos nominales y con cifras $(1,2,3 \ldots)$ los argumentos proposicionales; distinguimos los argumentos del predicado proposicional de los del principal añadiendo una coma alta: $a^{\prime}$, b', 1'...; los semas descritos figuran entre corchetes angulares y abreviados: $\langle$ pos $>$ : posibilidad, $<$ nec $>$ : necesidad, $\langle$ vol $>$ : volición; V: predicado principal, V': predicado proposicional; representamos entre corchetes [] los rasgos semánticos que deben caracterizar los argumentos nominales de un predicado; otras abreviaciones: I: infinitivo, prep: preposición, conj: conjunción..

2 aver (a) (de), convenir, desear, dever, dexar, estar, mandar, pedir, poder, prometer, querer, rogar, ser, tener, vedar.

${ }^{3}$ Denominamos cohesión el conjunto de propiedades sintáctico-semánticas que unen dos predicados en relación de complementariedad. Seguimos a Gómez Manzano (1991: 49) y a Henrichsen (1967: 49).
} 
infinitivo exige un actante $a$ [+humano], [+potente], mientras que en (3) $a$ es [-humano], [-potente]. En ambos casos, sin embargo, se trata de un caso lingüístico nominativo (o función sujeto):

(2) maguer fuyr queramos fazer non lo podemos PFG, 34v

(3) Bien dizen que la prudencia no puede ser sino en los viejos Cel, 133

- V no asigna caso lingüístico ninguno a $\mathrm{V}^{\prime}$ (el infinitivo no puede ser sustituido por un pronombre: lo),

- $\mathrm{V}^{\prime}$ 'es siempre un infinitivo,

$-a^{\prime}=a$,

— V puede describirse a partir de un solo sema modal de necesidad o posibilidad:

poder: $\mathrm{SN}_{\text {nom }}^{\mathrm{a}}<$ pos $>\mathrm{V}^{\prime}$, dever: $\mathrm{SN}_{\text {nom }}^{2}<$ nec $>\mathrm{V}$,

- algunos de estos verbos pueden presentar valores modales epistémicos junto a los factuales,

- V y V' ofrecen un alto grado de cohesión, lo que explica su carácter perifrástico.

\subsection{Predicados trivalentes: (a) mandar, vedar, dexar y (b) pedir, rogar,} prometer $V^{\prime}:$

— V asigna caso nocional y caso lingüístico a sus actantes, así como a

(4) hyo les mandare dar conducho mientra que por mi tierra fueren (PMC, 1356)

(5) e esto mando fazer el apostol sant jago e que lo fiziessen (Par, 13r)

(6) (los infantes de Carrión) ruegan al rey que los quite desta cort (PMC, 2989).

Con los verbos del grupo (a) (ejemplos (4) y (5)), a debe presentar los rasgos semánticos [+humano] [+autoridad], c [+humano], [-autoridad] y [+potente] respecto a $\mathrm{V}^{\prime}, \mathrm{V}^{\prime}$ puede ser un infinitivo o una proposición con que + subjuntivo.

En cambio, con el grupo (b) (ejemplo (7)), $a$ es [+humano] [-autoridad], $c$ [+humano], [+autoridad] y [+potente] respecto a $V^{\prime}, V^{\prime}$ solo puede presentarse como proposición que + subjuntivo,

$\longrightarrow$ y $c$ presentan propiedades semánticas correlativas y función sintáctica de sujeto para $a$ y de acusativo o dativo para $c$ (fluctuaciones con mandar, dexar y rogar),

$-\mathrm{V}^{\prime}=$ infinitivo / que +subjuntivo en alternancia libre con (a) y $\mathrm{V}^{\prime}=$ $q u e+$ subjuntivo con (b),

-presencia de un semema complejo para $\mathrm{V}$ : con el grupo (a): $<$ CAUSA $><$ DECIR $><$ MODAL $>$; con el grupo (b): $<$ DECIR $><$ MODAL $>$,

- valor exclusivamente factual para estos verbos (si exceptuamos prometer, que tendrá posteriormente al período estudiado valor epistémico), 
-módulos casuales propuestos:

(a) mandar: $\mathrm{SN}^{\mathrm{aAGEN}}{ }_{\text {nom }}<\mathrm{CAUS}><\mathrm{DECIR}><$ nec $>\left[\mathrm{V}^{\prime}\right]^{2 \mathrm{ACC}}{ }_{\text {acc }}$ a $\mathrm{SN}^{\mathrm{cDEST}}{ }_{\text {datacc }}$;

(b) rogar: $\mathrm{SN}^{\text {aNOM }}{ }_{\text {nom }}<\mathrm{DECIR}><\mathrm{nec}>\left[\mathrm{V}^{\prime}\right]^{2 \mathrm{ACC}}{ }_{\text {acc }}$ a $\mathrm{SN}^{\mathrm{cDEST}}{ }_{\text {datacc, }}^{\text {acc }}$

- cohesión débil entre $\mathrm{V}$ y V'.

\subsection{Predicados bivalentes: querer, desear, convenir...}

(7) e conviene al Rey seer asessegado e que non rria mucho (PP, 5v)

(8) Non quiero que nada pierda el Campeador (PMC, 1361)

(9) La guerra quiere costa e que non se duela de dar el que anda en ella (LM, 34r).

Estos verbos comparten propiedades con el tipo 2.1 y con el tipo 2.2:

Como 2.1:

$-a^{\prime}=a($ con querer, desear $)$,

- $\mathrm{V}^{\prime}$ 'asigna caso nocional a los actantes (convenir),

—un único sema para $\mathrm{V}$ : $<$ vol $>$ para querer y desear; $<\mathrm{M}>$, archilexema modal, para convenir,

—ambigüedad modal (factual, epistémica y axiológica) para convenir.

Como 2.2:

$-\mathrm{V}^{\prime}$ puede ser un infinitivo o una proposición con que + subjuntivo (en distribución libre con convenir (7), en distribución complementaria con querer $y$ desear (8),

-valor modal exclusivamente factual con querer y desear,

- V asigna caso nocional a sus actantes. Así, por ejemplo, cuando $a$ es [-humano], el valor de querer se ve modificado (9) y no expresa la volición.

3. Todos los grupos analizados comparten una misma característica: exigen, en su uso modal, un argumento proposicional. Las diversas soluciones sintácticas reflejarán un diferente grado de cohesión entre los dos predicados, V y V', como refleja el cuadro siguiente:

Cohesión entre $\mathrm{V}$ y V' con los diferentes predicados modales factuales:

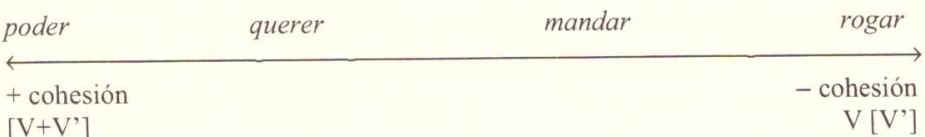

Destacaré dos aspectos de lo que acabamos de exponer:

- No hay verbos modales en sí, sino módulos actanciales en los que determinados verbos pueden desarrollar un sema modal.

-Dichos módulos deben comportar un doble nivel predicativo o, dicho de otra manera, una proposición. Sin embargo, si esta propiedad aparece estrechamente ligada al valor modal factual del verbo, no resulta suficiente para 
su caracterización, pues otros verbos de semántica no modal aceptan igualmente una estructura argumental proposicional.

Así pues, parece necesario ampliar el estudio a todos aquellos verbos que presentan un módulo proposicional con el fin de acotar mejor el grupo de los verbos modales y, en particular, de los verbos modales factuales.

4. Para esta segunda fase de mi trabajo, he comenzado con un corpus más reducido ${ }^{4}$, formado por obras en prosa y en verso, de naturaleza literaria o jurídica, de los siglos XI al XIII. En una segunda fase, en preparación, compararemos los resultados obtenidos con el funcionamiento del sistema latino, para, finalmente, ampliar el corpus con obras posteriores.

4.1. Procedimiento seguido: hemos considerado todas las apariciones de $\mathrm{V}$ seguido de proposición y hemos retenido las variaciones sintácticas siguientes: V + I, VprepI, VconjV'+ indicativo / subjuntivo. La conjunción más frecuente es que, pero hemos recogido también ejemplos con como y otros sin conjunción ninguna, en yuxtaposición. Por el contrario, no hemos considerado aquí (ya que, nos parece, necesitan un estudio aparte) las interrogativas indirectas, las estructuras de $\mathrm{V}+$ interrogativo + infinitivo (como no sé qué decir) y las relativas introducidas por lo que.

A partir de las tres posibilidades sintácticas anteriores, hemos establecido 14 clases sintácticas posibles:
1. VI
2. VprepI
3. VconjV'+ indicativo
4. VconjV'+ subjuntivo
5. VI en alternancia con VprepI
6. VI en alternancia con VconjV'+ indicativo
7. VI en alternancia con VconjV'+ subjuntivo
8. VprepI en alternancia con VconjV'+ indicativo
9. VprepI en alternancia con VconjV'+ subjuntivo
10. VconjV' con alternancia indicativo / subjuntivo
11. VprepI en alternancia con VconjV' + indicativo / subjuntivo
12. $\mathrm{VI} / \mathrm{VprepI} / \mathrm{Vconj \textrm {V }}$ ' + indicativo
13. VI/VprepI/VconjV'+ subjuntivo
14. VI / VprepI / VconjV'+ indicativo / subjuntivo.

\footnotetext{
${ }^{4}$ Documentos lingüisticos de España, Vol. 1. Reino de Castilla (s. XI y XII) (DLE), Auto de los Reyes Magos (1150 - 1200) (ARM), Disputa del Alma y el Cuerpo (1150 - 1200) (DAC), Siesta de Abril (1205) (SA), Vida de Santa Maria Egipciaca (1200 - 1215) (VSME), Fuero de Guadalajara (1219) (FG), Poema de Mio Cid (PMC), Poridad de las poridades (mediados del s. XIII) (PP).
} 
Una vez clasificadas sintácticamente cada una de las apariciones de verbos proposicionales, hemos constatado la existencia de ciertas regularidades semántico-sintácticas, como se muestra en el cuadro siguiente ${ }^{5}$ :

\begin{tabular}{|l|c|c|c|c|c|c|c|c|c|}
\hline & $\mathrm{C} 1$ & $\mathrm{C} 2$ & $\mathrm{C} 3$ & $\mathrm{C} 4$ & $\mathrm{C} 5$ & $\mathrm{C} 7$ & $\mathrm{C} 8$ & $\mathrm{C} 9$ & $\mathrm{C} 13$ \\
\hline $\mathrm{A}$ & 1 & 13 & & & & & & 1 & 1 \\
\hline $\mathrm{MF}$ & 2 & 2 & 2 & 9 & 2 & 3 & & 4 & 1 \\
\hline $\mathrm{MFv}$ & 2 & 3 & & 1 & & 1 & & & \\
\hline $\mathrm{MAx}$ & 2 & 3 & 5 & & & & & 1 & \\
\hline $\mathrm{P}$ & & & 1 & & & & & & \\
\hline Fc & & & & & & 1 & & & \\
\hline $\mathrm{ME}$ & & 1 & 13 & 2 & & & 1 & & \\
\hline $\mathrm{Mv}$ & 2 & 3 & & & 2 & & & & \\
\hline F & & 2 & & & 1 & & & & \\
\hline
\end{tabular}

De estos diferentes grupos, podemos ya apartar el de los verbos de movimiento y los finales (como dar $a+$ inf), puesto que la subordinada que los acompaña no tiene valor proposicional sino circunstancial final (no es, pues, un argumento obligatorio sino opcional).

5. Hemos observado la presencia de clases con alto grado de regularidad semántico-sintáctica:

\footnotetext{
${ }^{5}$ Donde A: aspectuales, MF: modales factuales, MFv: modales factuales volitivos, MAx: modales axiológicos, P: perceptivos, Fc: factitivos, ME: modales epistémicos, Mv: movimiento, F: finales.
} 


\subsection{Los verbos aspectuales}

\begin{tabular}{|l|c|c|}
\hline \multicolumn{1}{|c|}{ VERBOS } & Clase sintáctica & $\mathrm{N}^{\circ}$ de ejemplos \\
\hline soler & 1 & 8 \\
\hline acojerse a & 2 & 1 \\
\hline començar a & 2 & 12 \\
\hline començar de & 2 & 14 \\
\hline detardar de & 2 & 1 \\
\hline echarse a & 2 & 1 \\
\hline finar de & 2 & 1 \\
\hline fincar de & 2 & 1 \\
\hline husarse a & 2 & 1 \\
\hline husarse en & 2 & 1 \\
\hline meterse a & 2 & 2 \\
\hline prenderse a & 2 & 2 \\
\hline ser por & 2 & 1 \\
\hline tomarse a & 2 & 4 \\
\hline tornar a & 2 & 3 \\
\hline tornarse de & 2 & 1 \\
\hline penssar de & 9 & 19 \\
\hline penssar a & 9 & 1 \\
\hline dexar de / que & 13 & 6 \\
\hline lexar de & 13 & 81 \\
\hline TOTAL & & \\
\hline & & 1 \\
\hline
\end{tabular}

Este grupo ofrece representantes de la clase 1 (VI): soler, 2 (VprepI) y 9 (VI / VprepI / Vquesubj): dexar de, lexar de: en este último caso, el valor aspectual solo existe con la construcción VprepI. Lo mismo sucede con pensar $d e, a \ldots$ en el PP.

Observaciones sobre el módulo actancial de estos verbos:

- V' es siempre un infinitivo,

- V' determina la naturaleza semántica del sujeto, así según se trate de un verbo de acción o de sentimiento, tendremos un actante $a$ [+/-potente] (10) y (11):

(10) El que en buen ora nasco compeço de fablar (PMC, 1114)

(11) Moros e moras compeçaron de llorar (PMC, 856),

$-a^{\prime}=a$,

—alternancia de la preposición ( $a$, de ) no significativa. 


\subsection{Los verbos modales epistémicos}

\begin{tabular}{|l|c|c|}
\hline \multicolumn{1}{|c|}{ VERBO } & Clase sintáctica & $\mathrm{N}^{\circ}$ de ejemplos \\
\hline olvidar de & 2 & 1 \\
\hline asmar & 3 & 2 \\
\hline comedirse & 3 & 1 \\
\hline conosçer & 3 & 1 \\
\hline creer & 3 & 10 \\
\hline entender & 3 & 4 \\
\hline fiar & 3 & 2 \\
\hline firmar & 3 & 1 \\
\hline jurar & 3 & 8 \\
\hline otorgar & 3 & 2 \\
\hline pareçer & 3 & 1 \\
\hline semejar & 3 & 1 \\
\hline tener & 3 & 2 \\
\hline acordarse & 4 & 1 \\
\hline oir & 6 & 1 \\
\hline saber & 6 & 77 \\
\hline ver & 6 & 9 \\
\hline membrar de / que & 8 & 2 \\
\hline cuidar & 14 & 11 \\
\hline TOTAL & & 137 \\
\hline & & \\
\hline
\end{tabular}

Los ejemplos de la clase 6 presentan valor modal epistémico únicamente con la construcción que + indicativo. La construcción con infinitivo da lugar a valores semánticos diferentes: percepción (ver, oír), modal factual (saber, que expresa la capacidad o la posibilidad):

(12) Quando vio Mio Çid asomar a Minaya (PMC 919)

(13) Nunqua odi de homne deçir / que tant bona manera ouo en si (SA 84-5)

(14) otras tantas yeruas y auia / que sol no[m]brar no las sabria (SA 47-8)

(15) pedidas vos ha e rogadas el mio señor Alfonsso / atan fierme mientre e de todo coraçon / que yo nulla cosa nol sope dezir de no (PMC 2200-2).

Los ejemplos de la clase 8 alternan la construcción prepI / que + indicativo, sin cambio de significado.

(16) ...que nol membraua de morjr (VSME 94, 66r)

(17) Tanto quiere jugar et reyr, / quenol mjembra que ha de morjr (VSME 169 - 170 , $67 \mathrm{r})$.

Finalmente, la clase 14 (cuidar) presenta valor modal epistémico con las construcciones Vconj + indicativo / subjuntivo. Junto al infinitivo, el verbo expresa la intención, es decir, la modalidad factual volitiva: 
(18) En beuer et en comer et follia / cuydaua noche et dia (VSME 165-6, 67r)

(19) dizre vos lo que vi, nol uos i quedo fallir (DAC 2)

(20) et cada uno cuyda uençer mientra dura la lid (PP 55, 13)

(21) Cuydo que fuesse alguna antojança / ho alguna espantança (VSME 942-3, 76r)

(22) por çierto cuydo que es muerto (VSME 1355, 80r).

Observamos, pues, que existe un dominio casi total de la construcción V que + indicativo, con dos ejemplos de alternancia modal: (21) - (22), ligada al grado de certidumbre. Sin embargo, los módulos actanciales de los verbos epistémicos obedecen a tres modelos:

$\mathrm{SN}^{\mathrm{aNOM}}{ }_{\text {nom }}<\mathrm{creer} / \mathrm{saber}>\left[\mathrm{V}^{\prime}\right]^{2 \mathrm{ACC}}{ }_{\text {acc }}$, ordenados respecto a un eje de certidumbre (de lo categórico a lo dudoso),

$\left[\mathrm{V}^{\prime}\right]^{1 \mathrm{ACC}} \mathrm{acc} \mathrm{V}\left(\mathrm{a} \mathrm{SN}{ }^{\mathrm{bNOM}}{ }_{\text {dat }}\right)$ : membrar, olvidar / parecer, semejar,

$\mathrm{SN}_{\text {nom }}^{\mathrm{aAGEN}}<\mathrm{DECIR}><\mathrm{nec}>\left[\mathrm{V}^{\prime}\right]^{2 \mathrm{ACC}}{ }_{\text {acc }}\left(\mathrm{a} \mathrm{SN}^{\mathrm{cDEST}}{ }_{\text {dat }}\right)$ : jurar, firmar, otorgar, cuyo componente enunciativo $<\mathrm{DECIR}>$ les permite aparecer en enunciados performativos.

\subsection{Los verbos factitivos}

Solo hemos encontrado un verbo factitivo, fazer, lo que explica la alta homogeneidad semántico-sintáctica de esta clase:

\begin{tabular}{|l|c|c|c|}
\hline \multicolumn{1}{|c|}{ VERBOS } & Clase sintáctica & Obra & $N^{\circ}$ de ejemplos \\
\hline fazer & 7 & $\mathrm{SA}$ & 1 \\
\hline fazer & 7 & $\mathrm{FG}$ & 4 \\
\hline fazer & 7 & VSME & 5 \\
\hline fazer & 7 & PMC & 10 \\
\hline fazer & 7 & $\mathrm{PP}$ & 13 \\
\hline fazer que & 7 & $\mathrm{PP}$ & 1 \\
\hline TOTAL & & & 34 \\
\hline
\end{tabular}

La clase 7 ofrece dos construcciones: VI / Vque + subjuntivo. Hemos registrado un solo ejemplo para la completiva de subjuntivo (23).

(23) Alexandre, meted mientes en uestra fazienda toda uia, et sera uuestro conseio muy bueno, et uuestro fecho, et del meior ordenamiento que uos podredes fazer que non ayan miedo de uuestro escarmiento los buenos et los omnes de paz, et que uos teman los malos et los malfechores (PP 40, 14)

(24) Quando querie adentro entrar ariedro la fazien tornar VSME 70v (la = María).

El módulo actancial, aunque próximo al de los modales factuales como mandar, se debe sin embargo distinguir por la ausencia de componente enunciativo con fazer (López Izquierdo 2000b):

$\mathrm{SN}^{\mathrm{aAGEN}}{ }_{\text {nom }}<\mathrm{CAUS}>\left[\mathrm{V}^{\prime}\right]^{2 \mathrm{ACC}}{ }_{\text {acc }}\left(\mathrm{a} \mathrm{SN}^{\mathrm{CDEST}}{ }_{\text {dat }}\right)$ 


\subsection{Los verbos de percepción}

\begin{tabular}{|l|c|c|}
\hline \multicolumn{1}{|c|}{ VERBOS } & Clase sintáctica & $\mathrm{N}^{\circ}$ de ejemplos \\
\hline mirar & 3 & 1 \\
\hline oir & 6 & 16 \\
\hline ver & 6 & 30 \\
\hline TOTAL & & 47 \\
\hline
\end{tabular}

Grupo de alta regularidad, admite construcciones de clase 1 o de clase 3, pero en este caso los verbos presentan muy frecuentemente un deslizamiento hacia el valor modal epistémico, aunque no de manera obligatoria, como se constata en (25):

(25) et o vieredes que son mas flacos, y mandat ferir primera miente PP 56, 27. 


\subsection{Los verbos modales factuales}

\begin{tabular}{|c|c|c|}
\hline VERBOS & Clase sintáctica & $\mathrm{N}^{\circ}$ de ejemplos \\
\hline caler & 1 & 1 \\
\hline dever & 1 & 36 \\
\hline devedar de & 2 & 2 \\
\hline ser a & 2 & 6 \\
\hline ser de & 2 & 3 \\
\hline amenazar & 3 & 1 \\
\hline recabdar & 3 & 1 \\
\hline castigar & 4 & 4 \\
\hline conjurar & 4 & 2 \\
\hline conseiar & 4 & 1 \\
\hline establecer & 4 & 1 \\
\hline pedir & 4 & 1 \\
\hline perçebir & 4 & 2 \\
\hline seguir & 4 & 1 \\
\hline sobrelevar & 4 & 1 \\
\hline juzgar & 4 & 1 \\
\hline aver & 5 & 2 \\
\hline aver a & 5 & 31 \\
\hline aver de & 5 & 31 \\
\hline escusar & 5 & 1 \\
\hline escusar de & 5 & 3 \\
\hline mandar & 7 & 67 \\
\hline poder & 7 & 154 \\
\hline vedar & 7 & 2 \\
\hline convidar, convidar a & 9 & 3 \\
\hline $\begin{array}{l}\text { guardarse de, } \\
\text { guardar }\end{array}$ & 9 & 6 \\
\hline guisarse de, guisar & 9 & 2 \\
\hline rogar & 9 & 18 \\
\hline rogar de & 9 & 1 \\
\hline dezir & 10 & 6 \\
\hline convenir & 13 & 4 \\
\hline convenir que & 13 & 21 \\
\hline convenir de & 13 & 5 \\
\hline dexar & 13 & 9 \\
\hline TOTAL & & 430 \\
\hline
\end{tabular}


Grupo de baja regularidad semántico-sintáctica, con gran heterogeneidad de construcciones. Ha de tenerse en cuenta el mayor número de ejemplos encontrados, así como la abundante presencia de verbos que admiten más de una construcción sintáctica. La clase 3 (V que + indicativo) no es representativa, pues amenazar, como prometer, ofrece un comportamiento particular; el ejemplo con recabdar, por su parte, presenta un verbo modal en la subordinada. En estos casos, se neutraliza la oposición indicativo / subjuntivo:

(26) recabdado ha —como tan buen varon— / que del alcaçar una salir non puede (PMC, 2006-7).

Los verbos de la clase 5 ofrecen varias posibilidades de construcción sin cambio aparente de significado: aver (a) (de), convidar (a), escusar (de) + infinitivo.

(27) Tod ome que oviere a firmar ante alcaldes e jurados, primerament connonbre con quales firmara e digal sy quiere firmar o no e que firmas oviere a perçebir (FG

(28) Qui oviere de firmar a conçejo, firme con çinco de conçejo (FG 27).

Con los verbos de la clase 7 , alternan libremente el infinitivo y la proposición de subjuntivo. Sin embargo, con mandar y vedar, la construcción de infinitivo facilita una interpretación con identidad de sujetos $a=a^{\prime}$ :

(29) non vos tardedes, mandedes ensellar (PMC 317.

Con poder, los ejemplos presentan mayoritariamente el infinitivo. Dos casos con completiva deben leerse como estructuras elípticas $=$ si pudieredes (hacer) que...

(30) Et si pudieredes que sea uuestro escriuano el uestro alguazil, sera meior uestra fazienda et mas en poridat (PP 50,16).

(31) et si pudieredes que sean todas uestras cosas con arte, fazello (PP 57, 15).

En la clase 9, la alternancia de construcciones (VprepI / Vquesubj) con guardarse de / que - guisarse de / que no conlleva cambio semántico. Se ha encontrado un solo ejemplo con rogar de, con una preposición ambigua sintácticamente: nexo entre el $\mathrm{V}$ y el infinitivo o introductora de un infinitivo en aposición. Lo incluimos por tanto en la clase 4.

(32) Esto diziendo conpieçan la razon / lo quel rogava Alfonsso el del Leon / de dar sus fijas a los ifantes de Carrion (PMC 1926-8). tivo.

En la clase 10, dezir solo ofrece valor modal factual con que + subjun-

En la clase 13, convenir, con tres construcciones posibles (VI, VprepI, $\mathrm{V}$ que subj), no ofrece ninguna diferencia de sentido apreciable. Como hemos 
visto, convenir es un verbo de valor modal muy amplio, pues puede expresar tanto la modalidad factual como la epistémica y la axiológica. Su semema constaría pues de un sema modal $\langle\mathrm{M}\rangle$, indiferenciado.

En cuanto a dexar, solo tiene valor factual con VI (33), y valor aspectual en los otros casos (34) - (36):

(33) Dios dixo en que yo creo / dexame veyer lo que deseyo (VSME 79v)

(34) Mas non dexo hi de pecar, / ante comenco de peorar (VSME 415 - 6, 70r)

(35) Et de la franqueza es dexar omne que por las tachas encubiertas que son en los omnes que non demande nyn las quiera saber (PP 34, 17)

(36) Alexandre, non dexedes algunas uezes en el anno, dos o tres, que coman conuusco uestros privados et uestros ricos omnes et que ayan conuusco solaz (PP $38,21)$.

Según todo lo visto, la heterogeneidad sintáctica de los verbos modales factuales puede reducirse a dos construcciones: $\mathrm{V}+$ infinitivo (con presencia de preposición no significativa en la mayoría de los casos) / V que + subjuntivo, en alternancia libre.

A estas posibilidades sintácticas se les deben sumar los varios módulos actanciales que hemos visto en la primera parte.

\section{6. Los verbos modales factuales volitivos}

\begin{tabular}{|l|c|c|}
\hline \multicolumn{1}{|c|}{ VERBOS } & Clase sintáctica & $\mathrm{N}^{\circ}$ de ejemplos \\
\hline dessear & 1 & 1 \\
\hline gradar & 1 & 1 \\
\hline esforçar de & 2 & 1 \\
\hline puiar de & 2 & 1 \\
\hline punnar de & 2 & 1 \\
\hline punnar en & 2 & 4 \\
\hline sospirar & 4 & 1 \\
\hline querer & 7 & 189 \\
\hline penssar de & 9 & 1 \\
\hline penssar en & 9 & 1 \\
\hline pensar & 9 & 1 \\
\hline cuidar & 14 & 10 \\
\hline cuidar en & 14 & 213 \\
\hline TOTAL & & \\
\hline
\end{tabular}

Como los modales factuales vistos anteriormente, la heterogeneidad sintáctica de este grupo puede reducirse a dos posibilidades:

$\mathrm{V}+$ infinitivo (con o sin preposición): dessear, gradar, esforçar de, punnar de... Estos verbos están muy cerca de los aspectuales, incluso por su construcción de infinitivo preposicional / V que + subjuntivo: sospirar. 
Sin embargo, la alternancia de ambas construcciones no es libre, sino complementaria: esto y el sema $<$ vol $>$ presente en su semema es lo que nos permite diferenciarlos de los modales factuales.

\subsection{Los verbos modales axiológicos}

\begin{tabular}{|l|c|c|}
\hline \multicolumn{1}{|c|}{ VERBO } & Clase sintáctica & $\mathrm{N}^{\circ}$ de ejemplos \\
\hline amar & 1 & 3 \\
\hline amar & 1 & 1 \\
\hline mereçer & 1 & 1 \\
\hline osar & 1 & 5 \\
\hline osar & 1 & 9 \\
\hline trever & 1 & 1 \\
\hline quexarse de & 2 & 1 \\
\hline curiarse de & 2 & 1 \\
\hline fartarse de & 2 & 1 \\
\hline alabar & 3 & 1 \\
\hline alegrarse & 3 & 1 \\
\hline gradesçer & 3 & 2 \\
\hline gradir & 3 & 1 \\
\hline temer & 3 & 2 \\
\hline plazer de, que & 9 & 8 \\
\hline TOTAL & & 38 \\
\hline
\end{tabular}

Constituye el grupo menos homogéneo sintácticamente. Además, estos verbos no exigen un módulo proposicional para presentar el valor modal: adorar la música y adorar escuchar música son tan modales axiológicos el uno como el otro.

6. A partir de todo lo visto, podemos establecer una escala de regularidad semántico-sintáctica de los distintos grupos:

\begin{tabular}{lll} 
A, ME, Fact, P & MF, MFv & MAx \\
\hline+ & -
\end{tabular}

Hemos constatado la existencia de regularidades semántico-sintácticas en los verbos analizados, más acentuada con los contenidos aspectuales, modales epistémicos y los verbos de percepción.

Si aplicamos la noción de verbo proposicional para aquellos verbos que actualizan uno de sus semas solo en combinación con un módulo de doble nivel predicativo, tendremos que retener los grupos siguientes: 
-los aspectuales: VprepI

- los modales epistémicos: $\mathrm{V}$ que indic

-los modales factuales: VI - V que subj

-los factitivos: VI - V que subj.

La diferenciación de estos subtipos no podrá hacerse sin recurrir a los diferentes módulos actanciales en que intervienen: así, verbos muy próximos sintácticamente, como fazer y mandar, se distinguen gracias a sus módulos actanciales característicos.

Se puede igualmente concluir de todo lo visto que el valor de un verbo viene dado en gran parte por la estructura actancial con la que se combina. Es este el patrón que activa ciertos semas potenciales del verbo y neutraliza otros. Así, el sema modal de mandar no se actualiza en:

(36) Más amaria contigo estar que toda Espana mandar (SA 86),

porque la estructura actancial no es proposicional.

Según esto, el verbo selecciona sus argumentos, pero con frecuencia a partir de varias combinaciones actanciales posibles. Un mismo verbo puede así multiplicar sus significados gracias a los diferentes módulos casuales en los que entra a ocupar el lugar de predicado. Este recurso de máxima variación semántica con un mínimo de unidades formales se completa con la posibilidad que presentan ciertos verbos (los proposicionales) de introducir un actante proposicional en su módulo. Se sitúa así el significado del verbo en un nuevo nivel, más abstracto, capaz de modificar una acción o un ser, por medio de la actualización de un sema aspectual modal.

\section{BIBLIOGRAFÍA}

Gómez Manzano, P. (1992): Perifrasis verbales con infinitivo. Valores y usos en la lengua hablada. Madrid: U.N.E.D.

Henrichsen, A. J. (1967): "Les périphrases verbales du français moderne". En Revue Romane I, 45 - 56.

López Izquierdo, M. (2000a): Recherches sur la modalité. Les verbes de modalité factuelle en espagnol médiéval. Tesis de Doctorado, inédita. Universidad Paris X - Nanterre.

López Izquierdo, M. (2000b): "Semántica de las construcciones proposicionales en el primer romance castellano. Los verbos factitivos y los verbos modales factuales". En Actes du IXe Colloque de Linguistique Hispanique, en prensa.

Pottier, B. (1987): Théorie et Analyse en Linguistique. Paris: Hachette, 1992. 
Marta López Izquierdo

Pottier, B. (1992): Sémantique générale. Paris: P.U.F, 1992.

Tesnière, L. (1959): Élements de syntaxe structurale. Paris: Klincksieck, $1988^{2}$. 